\title{
LOS SERVICIOS EDUCATIVOS Y SANITARIOS EN EL DESARROLLO RURAL VALENCIANO: PRINCIPALES PROBLEMAS Y POSIBLES MEJORAS*
}

\author{
Jaime Escribano Pizarro \\ Dpto. de Geografía. Facultad de Geografía e Historia \\ Universidad de Valencia \\ Jaime.Escribano@uv.es
}

\begin{abstract}
Resumen: Se examinan, primero, los principales problemas que afectan al funcionamiento de los servicios educativos y sanitarios básicos en las zonas rurales, para permitir su uso por parte de la población local; y por otro, la propuestas concebidas por ésta para intentar resolver dichas dificultades, mejorar la prestación y calidad de estos, y poder integrar sus equipamientos en los procesos de desarrollo local. Al basarnos en una metodología cualitativa (entrevistas) y centrarnos en diferentes actores clave, los problemas y las soluciones señaladas se caracterizarán por su flexibilidad y multifuncionalidad. No obstante, no todas las respuestas podrán ser aplicadas a la vez, al requerir algunas de ellas bastante tiempo para su desarrollo.
\end{abstract}

Palabras clave: Servicios educativos, servicios sanitarios, espacio rural, LEADER+, Valencia.

\begin{abstract}
We examine, first, the main problems in operation of basic education and health-care services in rural areas to provide the local population efficiently; and second, the local population's suggestions of how to solve these difficulties, improve the provision and quality of the services, and integrate their equipment into local development processes. Having based the investigation on a qualitative methodology (interviews) and focusing on the local key actors, the indicated problems and solutions are characterised by flexibility and multifunctionality. However, not all offered solutions can be applied simultaneously, since some of them require time to be developed.
\end{abstract}

Keywords: Educational services, health services, rural space, LEADER+, Valencia.

* Recibido: 21-03-11 Aceptado: 23-06-11. 


\section{Servicios colectivos y desarrollo rural}

No es una novedad decir que los servicios son un componente vital de las economías nacionales y regionales, y que por tanto se presentan como el sector con el mayor impacto en términos de ocupación dentro de los países desarrollados (Melero y Calatrava, 2003). De hecho, a lo largo de las últimas décadas el estudio de los servicios desde el punto de vista geográfico ha evolucionado desde unas concepciones centradas casi exclusivamente en el empleo, la producción y el comercio, a otras en donde muchas de las nuevas investigaciones ya no son identificadas como trabajos sobre servicios en sentido estricto, ni siguen el camino trazado por generaciones anteriores (Tickell, 1999).

En la actualidad, nos encontramos ante un nuevo marco de análisis sobre los servicios, resultado de la progresiva evolución que presenta la sociedad actual, al pasar de unas necesidades humanas elementales a otras formas socioeconómicas de interacción y demandas más sofisticadas y complejas (Moreno y Escolano, 1992a; Tickell, 2002). En consecuencia, no sólo se consumen más servicios, sino que también su demanda se ha hecho mucho más diversificada (tanto en el tiempo como en el espacio); esta evolución ha favorecido que los servicios incrementen su relevancia y ambigüedad conceptual a la hora de aproximarnos a su definición y/o delimitación, y en última instancia, que el interés por explorar el desarrollo espacial y el impacto económico de los mismos permanezca aún como objeto pertinente (Illeris, 2004).

En este sentido, tradicionalmente el impacto de los servicios colectivos ${ }^{1}$ sobre el desarrollo de los municipios rurales, y en concreto el papel jugado por las actividades educativas y sanitarias, ha sido un tema objeto de numerosas investigaciones. Sobre todo, desde el punto de vista de la capacidad de las prestaciones derivadas de ambos servicios para atraer población y generar actividad económica (gracias a los ingresos de los "nuevos" residentes), en zonas donde la diversificación de la actividad productiva fuera compleja por deficientes condicionantes de localización y/o accesibilidad (Moreno y Escolano, 1992b; Peraldi y Pieri, 2006; Escalona y Díez, 2007).

Pero también, desde enfoques más centrados en aspectos sociales al hacer hincapié en la capacidad que tanto los servicios educativos como sanitarios tienen para, primero, ayudar a desarrollar la idea de pertenencia a un territorio, en la medida que los equipamientos de estos servicios constituyen puntos alrededor de los cuales los

\footnotetext{
${ }^{1}$ Según Moreno y Escolano (1992a) los servicios colectivos son aquéllos cuya finalidad es satisfacer las necesidades sociales, reproducir y mejorar la fuerza de trabajo, elevar el bienestar y la calidad de vida, y reproducir el estatuto social individual, a través de la participación de entidades públicas, privadas, mixtas y del Tercer Sector.
} 
individuos organizan sus espacios de vida de manera legible y coherente (Jean, 2007); y segundo, favorecer la formación y consolidación de las relaciones sociales como vía con la que poder contribuir al desarrollo local de un territorio dado ${ }^{2}$.

Este valor será, sobre todo, resultado del empleo que la población local haga de las prestaciones educativas y sanitarias como ámbitos de socialización a partir de los que expresar y compartir sus experiencias diarias, y en consecuencia, definir tanto sus necesidades como las respuestas más oportunas a las mismas. Es más, las relaciones que con frecuencia se generan alrededor de estos servicios, van a permitir disponer a menudo de todo un conjunto de reglas de confianza y vínculos basados en reciprocidades, que pueden terminar fácilmente por constituir sentimientos de pertenencia y voluntades de participación en la búsqueda de soluciones a los problemas propios y colectivos. De este modo, se presentan como un elemento clave para cualquier estrategia de desarrollo local (Subirats, 2002).

No obstante, Jean (1995) apunta que para que los servicios educativos y sanitarios básicos puedan aprovecharse por el territorio, como un activo capaz de favorecer procesos de desarrollo (a través sobre todo del capital social ${ }^{3}$ que pudieran generar), es necesario contar con la participación activa de la población local, los profesionales vinculados a la prestación y gestión de los servicios, las administraciones públicas y otros posibles actores del territorio (por ejemplo organizaciones culturales). Una premisa que en la actualidad, lejos de la evidencia de su necesidad, no siempre es posible de conseguir por muy diversos motivos.

De hecho, el primer objetivo del presente artículo es identificar y examinar el origen de los principales problemas que presenta el funcionamiento de los servicios educativos y sanitarios básicos en las zonas rurales, de cara a favorecer su prestación y permitir un uso más eficiente y eficaz por parte de la población local. Y en segundo lugar, recoger las vías de actuación posibles concebidas por la sociedad rural para intentar, si bien no resolver plenamente estas cuestiones problemáticas, al menos sí mitigarlas con el fin de mejorar la calidad del servicio, y así, aumentar las posibilidades de integrar sus equipamientos e instalaciones en los procesos de desarrollo local.

\footnotetext{
${ }^{2}$ Boix (2003) precisa más al señalar en relación a los servicios educativos, que una de las potencialidades de la escuela rural es precisamente su capacidad para ofrecer a sus alumnos y familiares en particular, pero también al conjunto de la sociedad en general, toda una serie de estrategias y recursos con los que poder entender y respetar la cultura local, valorar las fiestas tradicionales, el entorno natural, la propia historia del pueblo, etc.; es decir, ofrecer una estructura abierta a partir de la que crear, conservar y difundir conjuntamente todo una serie de conocimientos locales.

${ }^{3}$ Por capital social entendemos el conjunto de redes y las relaciones que tiene una persona con el resto de la sociedad, y que le van a permitir acceder a unos determinados recursos que, sin dichas interacciones no podría alcanzar (o al menos no al mismo "bajo" coste); por tanto, vemos que la cantidad de capital social de cualquier individuo dependerá de la cantidad de contactos de que disponga o sea capaz de establecer (Bourdieu, 1986).
} 


\section{Metodología: sujetos y área de estudio}

El ámbito de estudio seleccionado se corresponde con la delimitación ofrecida por la Iniciativa Comunitaria LEADER+ (2000-2006) de la Provincia de Valencia, ya que ésta encierra en su conjunto toda una serie de poblaciones capaces de ejemplificar los procesos de cambio demográfico, económico y productivo, políticos, de tipo sociocultural, y vinculados a las nuevas cuestiones de género que afectan actualmente a los territorios rurales españoles (Moyano, 2000; Romero y Farinos, 2007; Camarero, 2009).

La elección de los municipios concretos ha perseguido recoger los contrastes territoriales no sólo del medio rural seleccionado, sino también de la organización funcional de los servicios y su proyección en el territorio. Es por ello que los criterios utilizados han sido básicamente, por un lado, el tamaño de las poblaciones (figura 1); y por otro, la tipología de los establecimientos educativos y sanitarios básicos (figura 2).

Con el tamaño de las poblaciones se ha pretendido obtener las distintas argumentaciones que existen alrededor del papel que juegan los servicios señalados, puesto que en grandes líneas la actitud de la población beneficiaria no es la misma cuando las prestaciones derivadas de ellos están aseguradas físicamente en el mismo municipio de residencia (es decir, núcleos de tamaño relativamente elevado); su futuro se encuentra comprometido ante la falta de demanda suficiente (y por tanto, existen un elevado riesgo de desaparición); o directamente hay que recurrir a las instalaciones localizadas en zonas cercanas debido a su inexistencia local (con los consiguientes costes económicos y temporales que dicha opción conllevase), ante la escasa demanda de población municipal capaz de rentabilizar económicamente este tipo de prestaciones.

Por su parte, con la diferente tipología de establecimientos ${ }^{4}$ se ha pretendido recoger la mayor parte de situaciones en que se presentan y organizan los servicios educativos y sanitarios en el espacio local, ya que ni todos los municipios disponen del mismo grado de cobertura temporal, ni cuentan con la misma cartera de servicios. De este modo, los municipios analizados se han caracterizado por ser una muestra representativa de localidades con oferta directa educativa y sanitaria, o por presentar únicamente la oferta local de alguno de ellos.

${ }^{4}$ El sistema público valenciano de educación básica está formado por los Centros Educativos de Educación Infantil y Primaria, y los Colegios Rurales Agrupados (CRAS). Por su parte, el sistema público valenciano de atención sanitaria elemental presenta tres tipos de unidades: los Centros de Salud (centros de referencia para las demarcaciones territoriales mínimas, dotadas a su vez de servicio de urgencia); los Consultorios Médicos (idénticos a los anteriores, pero subordinados a ellos en cuanto a funciones); y los Consultorios Auxiliares (cuya función es apoyar a las unidades anteriores, presentando una menor dotación de recursos materiales y humanos). 


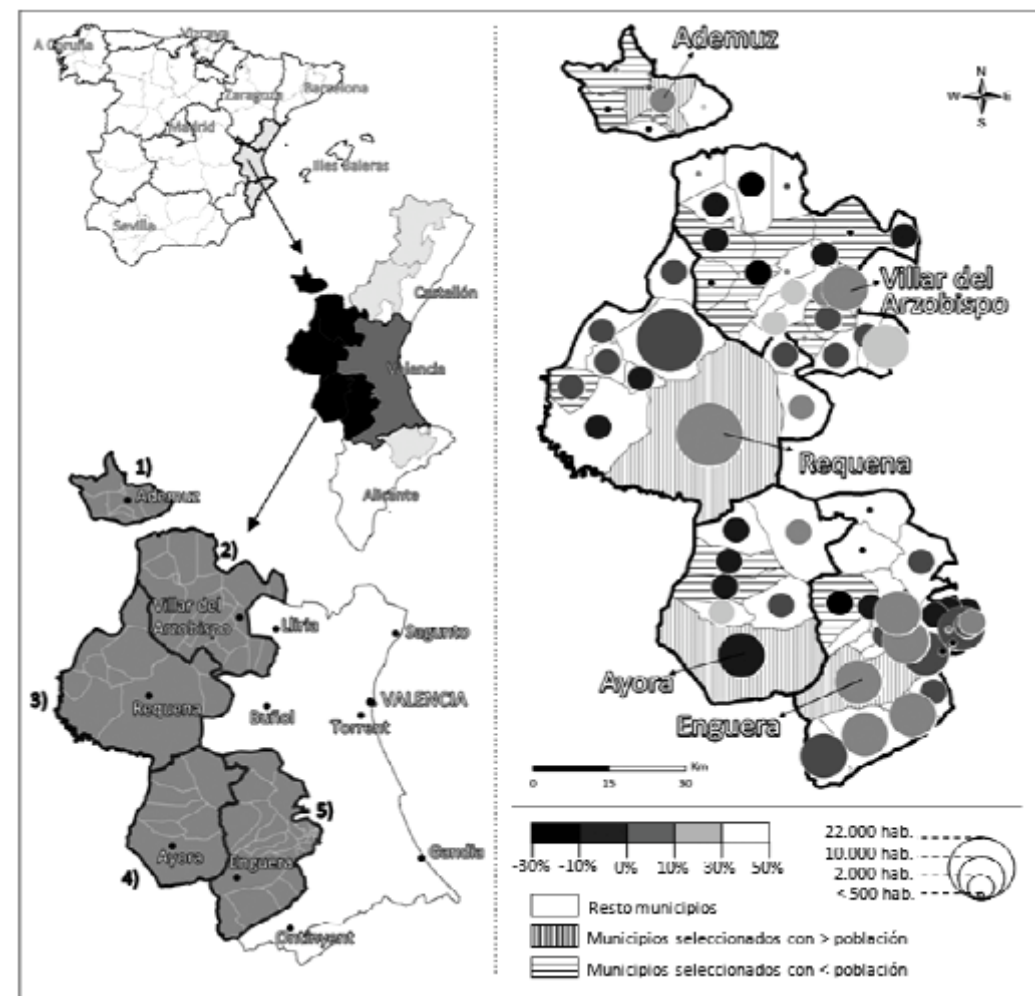

Figura 1. Municipios de estudio: evolución demográfica 2001-2010 y tamaño de población 2010.

Fuente: Elaboración propia a partir de la Unidad de Promoción y Animación del Desarrollo Rural, y los Grupos de Acción Local de los LEADER+ elegidos; y de los Censos de Población y Viviendas de 2001, y el Padrón Municipal de 2010 del INE (2011).

Nota: En la Provincia de Valencia podemos distinguir: 1) Rincón de Ademuz; 2) Serranía del Turia; 3) Tierras del Interior; 4) Valle de Ayora-Cofrentes; 5) Macizo del Caroig. El resto de grupos LEADER+ de la Comunidad Valenciana se reparten, como se puede ver en el mapa autonómico, dos en la Provincia de Castellón (al norte Els Ports-Maestrat, y al sur de éste limitando con la Provincia de Valencia el Alto Palancia-Alto Mijares) y uno más en la Provincia de Alicante (Aitana).

Una vez identificados los municipios, la información derivó de todo un conjunto de entrevistas personales dirigidas a diversos actores clave elegidos por su "amplia y contrastada" experiencia personal y/o profesional en estas zonas ${ }^{5}$ para, por una parte,

${ }^{5}$ Las entrevistas se realizaron entre los meses de abril y mayo de 2006, y de mayo a julio de 2007; la duración de las mismas osciló con frecuencia entre la hora y los tres cuartos de hora. Todas ellas fueron grabadas digitalmente en audio, previa solicitud de permiso al entrevistado, y transcritas posteriormente en 


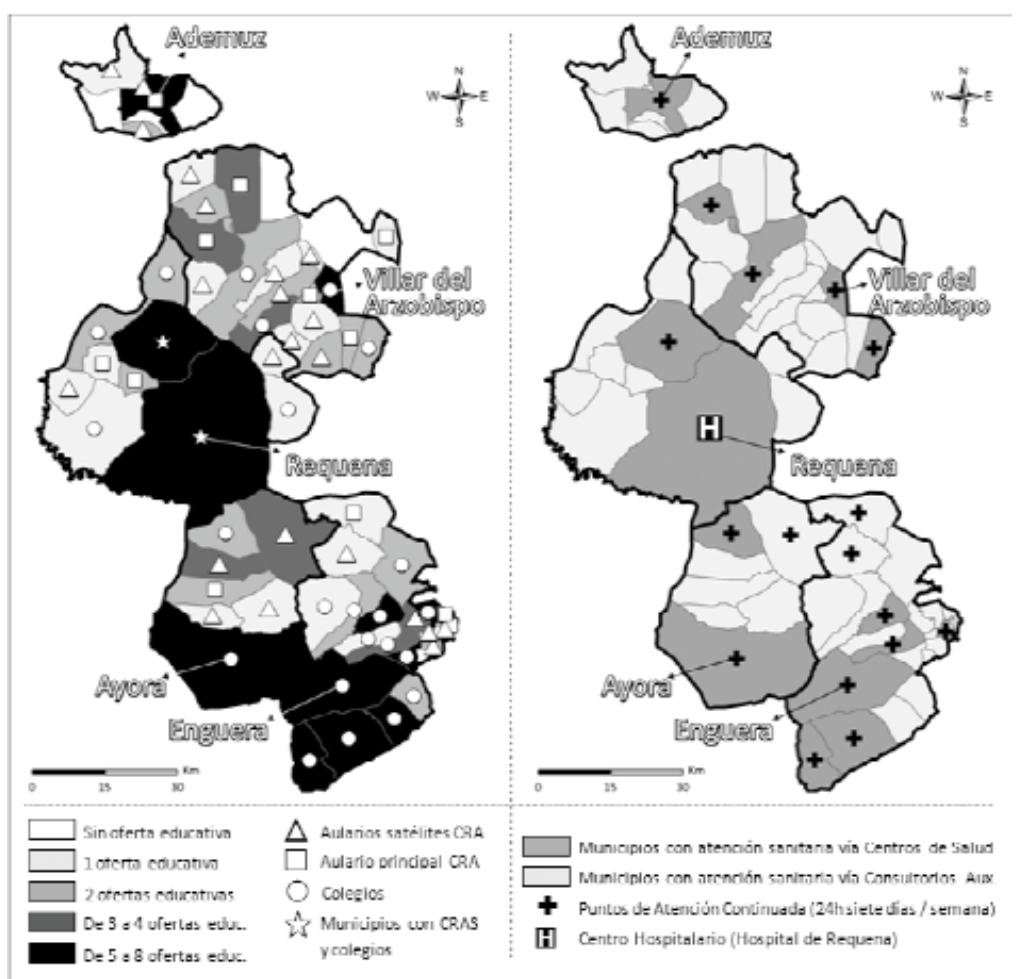

Figura 1. Municipios de estudio: evolución demográfica 2001-2010 y tamaño de población 2010.

Fuente: Elaboración propia a partir de la Guía de Centros Docentes de la Conselleria de Educació (Generalitat Valenciana), y del Listado de Centros de Atención Primaria de la Conselleria de Sanitat (Generalitat Valenciana).

Nota: Al hablar de oferta educativa entendemos opciones de educación que integran no sólo el nivel básico, sino también la educación postobligatoria (bachilleratos y módulos de formación profesional), educación infantil y pre-escolar, escuelas de música, idiomas, centros para adultos, etc. Recordemos que CRA equivale a decir Centro (de educación) Rural Agrupado.

su totalidad. Tal y como sugiere Valles (2002), desde el principio se planteó un análisis temático del conjunto de entrevistas, de manera que en primer lugar, el material se estructuró alrededor de los temas considerados más importantes (ver tabla 1); en segundo lugar, se realizó la codificación de los datos y el agrupamiento por categorías para, en una tercera fase, pasar a la interpretación de los discursos. Para ello, relacionamos lo expresado por los sujetos con procesos y elementos de la dinámica territorial presente en las zonas de estudio trabajadas, y pasamos a establecer comparaciones, similitudes y divergencias entre los dos grandes conjuntos de municipios-casos de estudio (es decir, entre aquéllos con mayor población, y los caracterizados por un tamaño demográfico menor). El objetivo no fue otro que identificar así, tendencias o modelos capaces de ser generalizables al conjunto de espacios rurales. 
ofrecer una visión integral del conjunto de problemas derivados de la disposición y organización espacial y funcional de los servicios elegidos; y por otra, albergar valoraciones fundamentadas sobre las posibles soluciones que cabría plantear y ejecutar de cara a potenciar el papel territorial de los equipamientos educativos y/o sanitarios.

De este modo, se realizaron un total de ochenta entrevistas ${ }^{6}$ : diecinueve a representantes políticos de las administraciones locales y supramunicipales; diecinueve más a los técnicos encargados de la puesta en marcha y/o gestión de los planes de desarrollo social y promoción económica; diecisiete a responsables de establecimientos sanitarios presentes en las zonas rurales elegidas; doce entrevistas a los directores, y jefes de estudio de los centros escolares con incidencia en los municipios seleccionados; y por último, trece más a miembros representativos de la sociedad local encargados de asociaciones relacionadas con el territorio, la sanidad, y/o la educación (presidentes de AMPAS, directores de escuelas de adultos, coordinadores de Protección Civil, etc.).

La elección de la entrevista personal como método de trabajo principal ${ }^{7}$, y en concreto la de tipo semiestructuado se debe: en primer lugar, por ser una de las herramientas más útiles y sencillas para obtener información comparable sobre un proceso tan amplio y plural como es el desarrollo local, en un ámbito territorial tan heterogéneo como es el medio rural (Esparcia, 2010). En segundo lugar, por ser una eficaz vía para recoger datos e información específica sobre los sistemas educativos y sanitarios básicos, de una manera actualizada y ajustada a la idiosincrasia espacial de estos medios, gran condicionante de su organización diaria. Y por último, un modo de trabajar práctico y útil para acceder a los comportamientos, motivaciones y valores con los que la sociedad rural actúa en relación a los servicios apuntados y bajo la perspectiva del desarrollo señalado.

Por medio de las variables de comportamiento se analizaron las distintas acciones llevadas a cabo respecto al desarrollo y mejora de la calidad ofrecida por estos servicios, así como la participación e interacción sociocultural de los mismos en la socie-

\footnotetext{
${ }^{6}$ Esta muestra forma parte del conjunto total de entrevistas realizadas para la elaboración de una Tesis Doctoral (financiada por el Programa de Formación Pre-doctoral "V Segles" de la Universidad de Valencia), enfocada a comparar el papel de los servicios públicos en los procesos de desarrollo local de diferentes áreas rurales de España y Francia.

${ }^{7}$ En todo caso, con el objetivo de mejorarla y darle un enfoque más eficiente, ésta metodología se acompañó en menor medida de otro conjunto de métodos cualitativos complementarios como son por ejemplo: la observación, el desarrollo de conversaciones informales (muchas veces fuera del lugar de las entrevistas), la realización personal e interpretación de fotografías de los espacios visitados, y el uso de diversas fuentes indirectas de tipo documental, como prensa nacional y/o regional; boletines de información local; periódicos escolares; páginas web de las áreas de estudio elegidas, de los centros educativos seleccionados, etc.; proyectos curriculares y actas de claustros; programaciones de AMPAS; memorias anuales de los Agentes de Empleo y Desarrollo Local; etc.
} 
dad local. Con el estudio de las motivaciones se planteó averiguar por qué se desarrollan unas determinadas acciones y no otras, y si éstas podían repetirse con facilidad en otros contextos similares. Y por último, con el examen de las valoraciones nos aproximamos a conocer primero, cuál es la opinión de los entrevistados sobre las actividades derivadas de los servicios y equipamientos educativos y sanitarios, y sus repercusiones en las dinámicas demográficas y económicas. Y en segundo lugar, saber qué ventajas e inconvenientes ofrece el medio rural a la organización de los servicios aquí presentados, y el grado con el que influyen sobre su calidad de vida.

Lógicamente, la información obtenida se apoyó en la interpretación y comparación de las respuestas de los entrevistados en relación con diversos conceptos y enunciados clave (agrupados normalmente por grandes temáticas). Entre ellos, los más importantes fueron, por un lado, aquéllos que nos permitieron conocer las acciones llevadas a cabo gracias a las prestaciones de los servicios analizados, y cómo éstas podían favorecer la participación local e integración sociocultural del municipio; y, por otro, los conceptos que nos facilitaban las opiniones de la población rural sobre la calidad ofrecida por estos servicios, y su capacidad para presentarse como elementos "condicionantes" de la evolución territorial de estas zonas (tabla 1).

\section{Principales problemas en la prestación de los servicios educativos y sanitarios en el medio rural valenciano}

En general, entre los diferentes motivos que explican la situación "desfavorable" en la que se encuentran ciertos núcleos rurales en términos de dotación de servicios públicos tenemos: primero, su menor volumen de demanda (no sólo en cuanto a cantidad de población, sino también por su elevada dispersión espacial); segundo, el mayor retraso al que se enfrentan en relación a la introducción y/o mejora de la calidad de las infra e infoestructuras; y por último, como resultado de su limitada influencia económica y/o política. En consecuencia, gran parte de la población residente en estas zonas termina por "aceptar" con frecuencia, no sólo una disminución de la escala de actuación de ciertos servicios (es decir, una reducción en términos de cantidad y/o naturaleza de la cobertura), sino también que determinadas ofertas no estén accesibles en las mismas condiciones que lo son para otros territorios (rurales o no), sobre todo en el caso de servicios especializados como hospitales, universidades... (Furuseth, 1998).

Aunque también es cierto que, la aceptación de esta situación se debe a otra serie de motivos más positivos, como son el aumento de la presencia y dotación de los equipamientos e instalaciones públicas (gracias sobre todo a la evolución normativa y legislativa experimentada en las últimas décadas, cada vez más sensible a las parti- 
Tabla 1. Conceptos rectores de las entrevistas realizadas.

\begin{tabular}{|c|c|c|}
\hline \multirow{4}{*}{ 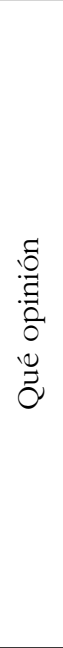 } & Concepto de calidad & $\begin{array}{l}\text { Calidad del servicio } \\
\text { Servicios como elementos de calidad de vida }\end{array}$ \\
\hline & $\begin{array}{l}\text { Caracterización de la influen- } \\
\text { cia de los servicios educativos } \\
\text { y sanitarios de carácter esen- } \\
\text { cial en la evolución de la } \\
\text { población y el poblamiento }\end{array}$ & $\begin{array}{l}\text { Como factor de retención frente a municipios de } \\
\text { orden similar o superior } \\
\text { Como factor de atracción frente a municipios de } \\
\text { orden similar o inferior }\end{array}$ \\
\hline & $\begin{array}{l}\text { Relaciones con los nuevos } \\
\text { contextos sociodemográficos }\end{array}$ & $\begin{array}{l}\text { Envejecimiento por dinámica natural e inmigratoria } \\
\text { (jubilados) } \\
\text { Frente a las nuevas corrientes inmigratorias (extran- } \\
\text { jeros jóvenes) }\end{array}$ \\
\hline & $\begin{array}{l}\text { Posibilidades de re-organiza- } \\
\text { ción en el territorio como ele- } \\
\text { mentos de mejora en la cali- } \\
\text { dad }\end{array}$ & $\begin{array}{l}\text { El papel de la nueva sociedad red (el tercer sector } \\
\text { y las organizaciones no lucrativas) } \\
\text { El papel de la TIC } \\
\text { Concentración vs. Dispersión espacial } \\
\text { El peso de los transportes }\end{array}$ \\
\hline \multirow{2}{*}{ 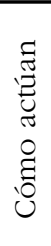 } & $\begin{array}{l}\text { Articulación con el resto de la } \\
\text { sociedad desde el punto de } \\
\text { vista "institucional" }\end{array}$ & $\begin{array}{l}\text { Programas conjuntos } \\
\text { Uso de equipamientos e instalaciones }\end{array}$ \\
\hline & $\begin{array}{l}\text { Articulación con el resto de la } \\
\text { sociedad desde el punto de } \\
\text { vista "personal" }\end{array}$ & $\begin{array}{l}\text { Preferencias por lugar de trabajo y residencia } \\
\text { Relación con asociaciones y/o ejecución de iniciati- } \\
\text { vas personales }\end{array}$ \\
\hline
\end{tabular}

Fuente: Elaboración propia.

cularidades del medio rural), y la mejora acontecida recientemente en términos de movilidad, accesibilidad y proximidad a los servicios en cuestión (Rodríguez González, 1999; Entrena, 2006).

No obstante, como apuntan los entrevistados, todavía queda margen de mejora en la prestación de los servicios educativos y sanitarios básicos. Por un lado, en función de la ineficiente utilización de los recursos disponibles; y por otro, según las necesidades e insatisfacciones derivadas en parte, y paradójicamente, de la organización y funcionamiento actual de prestaciones educativas y sanitarias (en tanto en cuanto algunas de las reformas introducidas no atendieron plenamente los problemas que pretendieran resolver, al enfatizar más la búsqueda de resultados inmediatos que desarrollar modificaciones estructurales).

En consecuencia, muchas veces nos encontramos en realidad no solo con el mantenimiento (e incluso refuerzo) de ciertos problemas "tradicionales", sino que también 
se observa la aparición de otros nuevos, como veremos a continuación. Lógicamente, las situaciones más problemáticas son más habituales en aquellos municipios en donde la infraestructura y dotación de uno o de ambos servicios es más restringida en cuanto a cantidad, tipología y capacidad de atención. Por consiguiente, es normal que la población de estas localidades experimente con frecuencia los problemas relacionados con los servicios públicos con una mayor intensidad.

En general, siete son los problemas identificados de forma recurrente y con similar grado de relevancia por la sociedad rural, en relación a la organización y disposición que presentan los servicios básicos de educación y sanidad en el espacio: a) falta (o ausencia) de coordinación entre administraciones y entre las entidades encargadas de la prestación de los propios servicios; b) reducida cobertura temporal (o incumplimiento de las franjas horarias establecidas); c) escasez de personal (y saturación de éste por el número y tipo de tareas a realizar); d) cierta inestabilidad y desmotivación de las plantillas por permanecer en estas zonas, junto a la pérdida que conlleva en cuanto a calidad de servicio y opciones de desarrollo local; e) ausencia de apoyos amplios y continuos por parte de la sociedad local para iniciar y/o mantener iniciativas favorables tanto para los servicios como para la propia población; f) dificultades para asegurar la oferta básica en los "márgenes del servicio"; y por último, g) necesidades constantes de actualización y adaptación a los cambios sociales (envejecimiento, emancipación de la mujer, digitalización de los servicios, etc.).

El primero de ellos hace referencia a la necesidad de introducir una mayor y mejor coordinación alrededor de las prestaciones educativas y sanitarias públicas, desde un doble punto de vista: por un lado, territorialmente entre espacios rurales y urbanos; y por otro, de un modo sectorial, resultado de las relaciones que los servicios educativos y/o sanitarios muestran con administraciones locales (tanto cuando éstas actúan de forma independiente, como cuando se agrupan en entidades supramunicipales):

a) En el primer caso, se trata de mejorar la relación de programas y actuaciones entre los niveles elementales de los servicios educativos y sanitarios presentes en estas zonas, y los servicios especializados vinculados a los mismos servicios pero existentes en áreas urbanas próximas. En definitiva, se trata por ejemplo de asegurar y/o aumentar la coordinación en los servicios de traslado pacientes y/o sus expedientes entre diferentes tipos de unidades de atención sanitaria (centros de salud y hospitales); organizar de manera conjunta los horarios laborales de las plantillas compartidas, una circunstancia habitual debido a que el aumento de la cartera de servicios en los centros de salud rural sólo se ha conseguido gracias a la presencia deslocalizada en ellos de especialistas procedentes de unidades hospitalarias o centros de especialidades urbanos cercanos; etc. 
b) En el segundo caso, se denuncia la falta de coordinación entre los servicios educativos y/o sanitarios y la propia administración local de estas zonas, a la hora de por ejemplo, plantear soluciones conjuntas ante problemas concretos de dotación de recursos humanos y/o materiales. En este sentido, puesto que los entrevistados señalan que pocas veces hay comunicación entre los ayuntamientos y los centros educativos y sanitarios, no es inusual encontrar propuestas de mejora por parte de las administraciones locales que no fructifican porque se desconocen en realidad las necesidades sociales, o simplemente porque no se atienden las opiniones de los profesionales encargados de dichos servicios, y se plantean por tanto de forma unilateral.

Junto a éste, otro problema relacionado con la administración local es su "incapacidad" para desarrollar actuaciones de forma conjunta con sus homólogos vecinos, como resultado de enfrentamientos por alcanzar una mejor renta de situación respecto a posibles competidores del entorno (por ejemplo, en cuanto a la obtención de recursos que les hicieran territorialmente más atractivos, caso de las inversiones para nuevos equipamientos, etc.) (Saco, 2010).

El segundo problema identificado deriva de la necesidad de asegurar y ampliar la atención ofrecida por ambos sistemas de servicios en sus niveles más elementales, especialmente en cuanto a cobertura temporal. Básicamente, porque de poco o nada sirve contar con una amplia red espacial de equipamientos e instalaciones si la población usuaria no puede hacer uso de ellos ni tan siquiera en su horario de referencia, por ausencia del personal responsable del servicio al concentrarse éste en los centros con mayor demanda, como medida con la que rentabilizar unos recursos humanos reducidos.

Obviamente, se trata de una cuestión que afecta además de a los usuarios de estos servicios, a los propios profesionales educativos y/o sanitarios; de hecho, la escasez de personal es otro de los problemas más comúnmente señalados por todo tipo de actores. No sólo porque contar con plantillas reducidas conlleva a que los trabajadores disponibles deban hacer frente a numerosas responsabilidades (algunas incluso fuera de sus propias competencias), sino que igualmente ante situaciones de ausencia de los responsables titulares de estos servicios por enfermedad o descanso, o incluso por cuestiones de agrupación de la demanda como ocurre con determinadas especialidades sanitarias (caso de la pediatría), ciertos profesionales terminan por trabajar de forma "simultánea" en varios centros (distantes por varias decenas de kilómetros), y abarcar así grandes extensiones de espacio al cabo de la jornada laboral. En general, esta situación es percibida como una importante desventaja frente a entornos urbanos, en donde la disponibilidad de profesionales es más amplia y se consigue, habitualmente, en un menor número de días. 
En cuarto lugar, siguiendo con los profesionales educativos y sanitarios (en este caso, más como "responsables" que como afectados), otro problema detectado por la sociedad local hace referencia a su escasa estabilidad laboral y, por consiguiente, al deterioro de la calidad y reducido aprovechamiento integral que se obtiene de los servicios y equipamientos localizados en las zonas rurales. El origen de esta situación se encuentra, como denuncian los entrevistados, en el uso recurrente que el personal laboral de estos servicios hace de los establecimientos situados en los núcleos rurales como medio para alcanzar otros destinos más atractivos y deseados (a través de la acumulación de puntos y méritos válidos en concursos de traslados). Este resultado deriva paradójicamente de la estrategia utilizada por la administración regional para hacer más atractiva la labor profesional en estas zonas (con frecuencia rechazadas en las solicitudes de emplazamiento laboral), al recompensar los costes que supusiera para ciertos profesionales trasladarse diariamente hasta esta zonas para desempeñar su trabajo.

En consecuencia, es habitual que las plantillas de ambos servicios presenten cierta inestabilidad crónica, y que ésta afecte a la calidad de la oferta prestada si tenemos en cuenta que gran parte de los establecimientos se ven abocados a reiniciar regularmente su organización, alterándose así la eficacia del servicio y la propia satisfacción de sus usuarios; en especial, porque muchos de estos "recién llegados" presentan una cierta desmotivación como consecuencia de tener que trabajar en emplazamientos no deseados, con además nulos conocimientos sobre el funcionamiento y organización habitual no sólo de estos servicios en medio rural, sino también de la casuística particular de estos territorios. Esta situación deriva en gran parte de la ausencia o escasa preparación específica que estos profesionales reciben durante su formación universitaria (Rubio, 2003; Morente, 2004).

Pero además, asociada a esta inestabilidad y desmotivación, otra cuestión problemática es la nula o limitada participación de este tipo de personal en actuaciones extraprofesionales que supusieran cierta inversión de tiempo ${ }^{8}$. Es así que la mayor parte de la atención complementaria existente, es resultado de programaciones de reducida amplitud (temporal y/o social), apoyadas en acciones puntuales, planificadas y promovidas de antemano por niveles jerárquicos superiores (externos además a estas zonas), y que en caso de realizarse, pudieran acompañarse de compensaciones económicas y/o curriculares. En consecuencia, con frecuencia los equipamientos e infraestructuras educativas y sanitarias disponibles no son utilizados al máximo de sus posibilidades (entendiendo este uso desde un enfoque integral), al quedar relegadas tan sólo a ejercicios de carácter obligatorio y relativamente excepcional.

\footnotetext{
${ }^{8}$ Aunque esto no siempre es así. Morales (2006) señala que en otras ocasiones la participación extraprofesional no es más amplia porque se encuentran: a) relativamente aislados, al no coincidir con actores locales que les apoyen; b) con la dificultad de recurrir a recursos suplementarios debido a los numerosos trámites burocráticos; y c) sin tejido social estructurado y motivado.
} 
En este sentido, se desaprovecha un valioso referente para los procesos de desarrollo local; básicamente, porque se trata de unos profesionales que por su papel en la sociedad rural podrían convertirse con relativa facilidad en promotores de espacios favorables a la participación comunitaria, gracias a su formación y preparación, su posición destacada en la comunidad (al recibir una cantidad significativa de diagnósticos territoriales de los propios habitantes), y su relativa capacidad para contactar con entidades y organismos externos capaces de aportar recursos (Díez, 1989; Santamaría, 1999).

En quinto lugar, la ausencia de apoyo por parte de la sociedad local también puede convertirse en un serio problema. No cabe duda que en el medio rural el respaldo de la población (tanto individual como colectivamente, como por ejemplo a través de las AMPAS), adquiere una trascendencia mucho más importante que en zonas urbanas. El mejor ejemplo lo encontramos en los servicios educativos, en donde precisamente los padres son las principales responsables de que una escuela rural se mantenga o no abierta, ya que en definitiva está en su mano la decisión del centro al que acudirán sus hijos (Morales, 2006). Aunque con frecuencia su capacidad de actuación es mucho mayor, gracias a que cuando trabajan de forma agrupada, en primer lugar, se constituyen a menudo en un apoyo financiero clave a través de los recursos que obtienen vía actividades sociales como sorteos, comidas, etc.; en segundo lugar, suponen una ayuda inigualable para la organización y gestión de actividades educativas y/o extra-educativas (preparación de salidas, distribución de material escolar, comedor y transporte escolar, etc.); y en tercer lugar, tienen cierta capacidad para ejercer en ocasiones, la suficiente presión social como para evitar la supresión de puestos docentes, la reducción de clases, etc. (Orriols, Roldán y Oliart, 2004; Poirey, 2006).

En consecuencia, no es de extrañar que la falta de un apoyo social, si bien no amplio, al menos sí constante en el tiempo, sea otro de los problemas identificados por los entrevistados. Esto es así sobre todo porque en la mayoría de casos, tan sólo se cuenta con apoyo reducido y puntual, y esto a su vez es resultado más de aspectos concretos de carácter problemático (por ejemplo, vinculado a las evaluaciones educativas de los alumnos) que consecuencia de una verdadera implicación constante y activa, que supere la simple adhesión retórica a cualquier tipo de iniciativa y por tanto, revierta la habitual escasa o nula participación (esperando así que las actividades las hagan otros). De hecho, la falta de movilización real de los integrantes de las asociaciones vinculadas a estos servicios, hace que con frecuencia éstas resulten poco atractivas en su composición actual.

En sexto lugar, la progresiva demanda por incrementar y perfeccionar la accesibilidad a la oferta presentada por los equipamientos educativos y sanitarios en los "márgenes del servicio", es señalada como otro de los problemas más repe- 
tidos. Es decir, se plantea la conveniencia de actuar en aquellas necesidades de la población que quedan contempladas en las ofertas y atenciones estipuladas como básicas, pero que por su carácter de relativa excepcionalidad en cuanto a la recurrencia con que se presentasen, registraran una estructuración comparativamente menor y/o más reducida en estas zonas frente a otras urbanas (por ejemplo, el transporte de urgencia en ambulancia a centros hospitalarios, o determinadas atenciones educativas a alumnos con necesidades especiales). De este modo, se podría evitar que el medio rural continuase presentando una posición de desventaja en ciertos aspectos esenciales, al depender de los recursos y ofertas especializadas que se encuentran en otras zonas más y mejor dotadas desde dicho punto de vista, simplemente por concentrar mayores niveles de demandas "excepcionales" de este tipo de atenciones. Este hecho no es sólo negativo por establecer una relación de dependencia en ocasiones muy intensa, sino también porque conlleva a que la eficacia en el uso de dichos recursos sea relativamente baja, debido a las deficientes condiciones de accesibilidad espacio/temporal que desde el medio rural hay todavía a estos (tabla 2).

En séptimo lugar, la creciente necesidad de acondicionar las atenciones educativas y sanitarias rurales a los nuevos contextos sociales y demográficos (emancipación sociolaboral femenina, envejecimiento, etc.), y a las incipientes formas de consumo de estos servicios (especialmente a través de las TIC), constituye en conjunto el último ámbito de problemas identificados por los entrevistados:

- En el primer caso, el mejor ejemplo deriva del envejecimiento progresivo que caracteriza al medio rural, y que plantea a su vez dos tipos de demandas: por un lado, ante la oportunidad que supone introducir determinadas prestaciones especialmente dirigidas a estos grupos de usuarios, como secciones de geriatría, o el incremento de las actuaciones desarrolladas por parte de los fisioterapeutas (en cuanto a trabajos de rehabilitación). Y por otro, resultado de la necesidad cada vez mayor de mejorar el sistema de transporte sanitario destinado al traslado habitual entre domicilios y establecimientos sanitarios, de la población mayor sin medio de desplazamiento.

- En el segundo caso, las peticiones para acondicionar las prestaciones educativas y sanitarias van encaminadas sobre todo a conseguir las infraestructuras y equipamientos necesarios con los que poder hacer frente al aumento de las formas de gestión y comunicación digitales. De este modo, se conseguiría por ejemplo mejorar el tratamiento de la información de los usuarios; incrementar la coordinación entre los miembros de los CRAS; etc.

Identificados los principales problemas, las soluciones más atractivas y posiblemente eficaces que podemos aportar, parten de los propios entrevistados. Básicamente, porque por su vinculación laboral y residencial con el medio rural, y su 


\begin{tabular}{|c|c|c|c|c|c|c|c|c|c|}
\hline \multirow{2}{*}{$\begin{array}{c}\text { Servicios sanitarios } \\
\text { Departamento } \\
\text { Arnau Vilanova }\end{array}$} & \multirow[b]{2}{*}{$\begin{array}{l}\text { Localiza- } \\
\quad \text { ción }\end{array}$} & \multicolumn{8}{|c|}{$\begin{array}{c}\text { Municipio de estudio (Territorios LEADER+ Rincón de Ademuz } \\
y \text { Serranía del Turia) }\end{array}$} \\
\hline & & $\begin{array}{l}\text { Ade- } \\
\text { muz }\end{array}$ & $\begin{array}{l}\text { Castiel- } \\
\text { fabib }\end{array}$ & $\begin{array}{l}\text { Casas } \\
\text { Altas }\end{array}$ & Tuéjar & Chelva & $\begin{array}{l}\text { Sot de } \\
\text { Chera }\end{array}$ & Andilla & $\begin{array}{c}\text { Villar } \\
\text { del } \\
\text { Arzo- } \\
\text { bispo }\end{array}$ \\
\hline Centro de especialidades & Burjassort & 112 & 126 & 112 & 69 & 61 & 66 & 67 & 46 \\
\hline Centro sanitario integrado & Lliria & 119 & 133 & 113 & 51 & 43 & 47 & 49 & 27 \\
\hline Centro de salud pública & Valencia & 115 & 130 & 116 & 81 & 73 & 77 & 83 & 57 \\
\hline & Chelva & 67 & 91 & 61 & 9 & 0 & - & - & - \\
\hline Unidad de rehabilitación & Villar del & & & & & & & & \\
\hline & Arzobispo & - & - & - & - & - & 31 & 41 & 0 \\
\hline $\begin{array}{l}\text { Unidad de conductas } \\
\text { adictivas }\end{array}$ & Paterna & 109 & 123 & 110 & 70 & 62 & 66 & 68 & 47 \\
\hline $\begin{array}{l}\text { Unidad de odontología } \\
\text { preventiva }\end{array}$ & Lliria & 119 & 133 & 113 & 51 & 43 & 47 & 49 & 27 \\
\hline Unidad de prevención & & & & & & & & & \\
\hline de cáncer de mama & Burjassot & 112 & 126 & 112 & 69 & 61 & 66 & 67 & 46 \\
\hline $\begin{array}{l}\text { Planificación familiar } \\
\text { Servicios de información }\end{array}$ & Lliria & 119 & 133 & 113 & 51 & 43 & 47 & 49 & 27 \\
\hline y atención al paciente & Burjassot & 112 & 126 & 112 & 69 & 61 & 66 & 67 & 46 \\
\hline Unidades de salud mental & Lliria & 119 & 133 & 113 & 51 & 43 & 47 & 49 & 27 \\
\hline Hospital(es) & & & & & & & & & \\
\hline Arnau de Vilanova & Valencia & 115 & 130 & 116 & 81 & 73 & 77 & 83 & 57 \\
\hline Obispo Polanco & Teruel & 44 & 46 & 46 & - & - & - & - & - \\
\hline
\end{tabular}

Fuente: Elaboración propia a partir de los datos de la Organización Asistencial de la Conselleria de Sanitat, 2011; y Via Michelín, según la opción de itinerario más rápido. Consultas realizadas el 19/05/2011.

Nota: Tanto el territorio LEADER+ del Rincón de Ademuz como de la Serranía del Turia pertenecen en su totalidad al Departamento Sanitario regido por el Hospital Arnau de Vilanova (situado en uno de los acceso norte de la ciudad de Valencia). En cuanto a la localización de los estos centros sanitarios, sólo se señalan los más cercanas (en tiempo) de toda la oferta que hay en cada caso. Por ejemplo, de las dos Unidades de Conductas Adictivas posibles (Paterna y Moncada), se señala la más próxima (Paterna). En el caso de los municipios del Rincón de Ademuz (Ademuz, Castielfabib y Casas Altas), existe un acuerdo interprovincial para que sus poblaciones puedan usar al Hospital de Teruel.

experiencia diaria como usuarios y/o responsables de dichos servicios, hacen difícil encontrar otras valoraciones y planteamientos que afronten de raíz los problemas señalados.

\section{Propuestas de actuación para mejorar las prestaciones educativas y sanitarias}

En líneas generales, las vías de actuación más repetidas con las que mejorar algunos de los principales problemas antes enunciados, quedan agrupadas en una serie 
de grandes bloques marcados por su relativa interconexión; sobre todo, si tenemos en cuenta que incluyen diversas acciones que permitirán abordar de forma simultánea diferentes cuestiones y/o deficiencias (figura 3). Esta multifuncionalidad se observa además, como un aspecto atractivo, puesto que con un conjunto relativamente reducido de propuestas se pueden abordar una amplia serie de demandas insatisfechas.

En primer lugar, siguiendo el orden de problemas expuestos anteriormente, destaca la necesidad por alcanzar una mayor y mejor coordinación entre los diferentes actores con incidencia en el medio rural, independientemente de que su capacidad de actuación sea de ámbito local y/o regional. Para ello, se señalan dos medidas a adoptar: primero, vinculadas a la superación (y eliminación) de toda una serie de barreras implícitas a la cooperación; y segundo, generalizar determinadas actuaciones dirigidas al trabajo en común, puestas ya en marcha por algunos de los entrevistados, y destacadas por sus buenos resultados.

a) Con relación al primer aspecto, se hace hincapié en el beneficio que puede conllevar la superación, por un lado, de las tradicionales actitudes de confrontación entre localidades vecinas, heredadas de enfrentamientos pasados y que en definitiva impiden la puesta en marcha de actuaciones conjuntas que, de otro modo, no pueden llegar a plantearse (por su elevado coste), o que en el caso de ejecutarse ven reducida su productividad y, por tanto, su propio mantenimiento. Y por otro, evitar planteamientos unilaterales $\mathrm{y} / \mathrm{o}$ individualistas a la hora de realizar actuaciones con cierto impacto territorial, con el fin de obtener una posición privilegiada en el acceso a determinados beneficios y/o reconocimientos económicos, sociales o políticos.

b) Paralelamente, los métodos de trabajo señalados por su utilidad para mejorar y ampliar la coordinación entre los diferentes territorios rurales y sus respectivos actores, se organizan alrededor de dos ideas básicas:

- Por un lado, conservar los canales de coordinación que en la actualidad muestran ya cierto éxito (por reducido que éste sea), aun cuando estos son tan sólo resultado de cuestiones ocasionales, secundarias y/o que sólo afectan a segmentos muy concretos de la sociedad local. El motivo no sería otro que servir de ejemplo sobre la actitud que cabe adoptar de cara a solucionar problemas comunes.

- Y por otro, apoyar la creación de estructuras de encuentro "multinivel", con las que conseguir espacios de difusión y discusión en los que agrupar a la mayor diversidad y número de actores locales. De este modo, y siempre contando con la voluntad de los interesados, se pueden comenzar a desarrollar labores de coordinación mediante la puesta en común y organización de acti- 


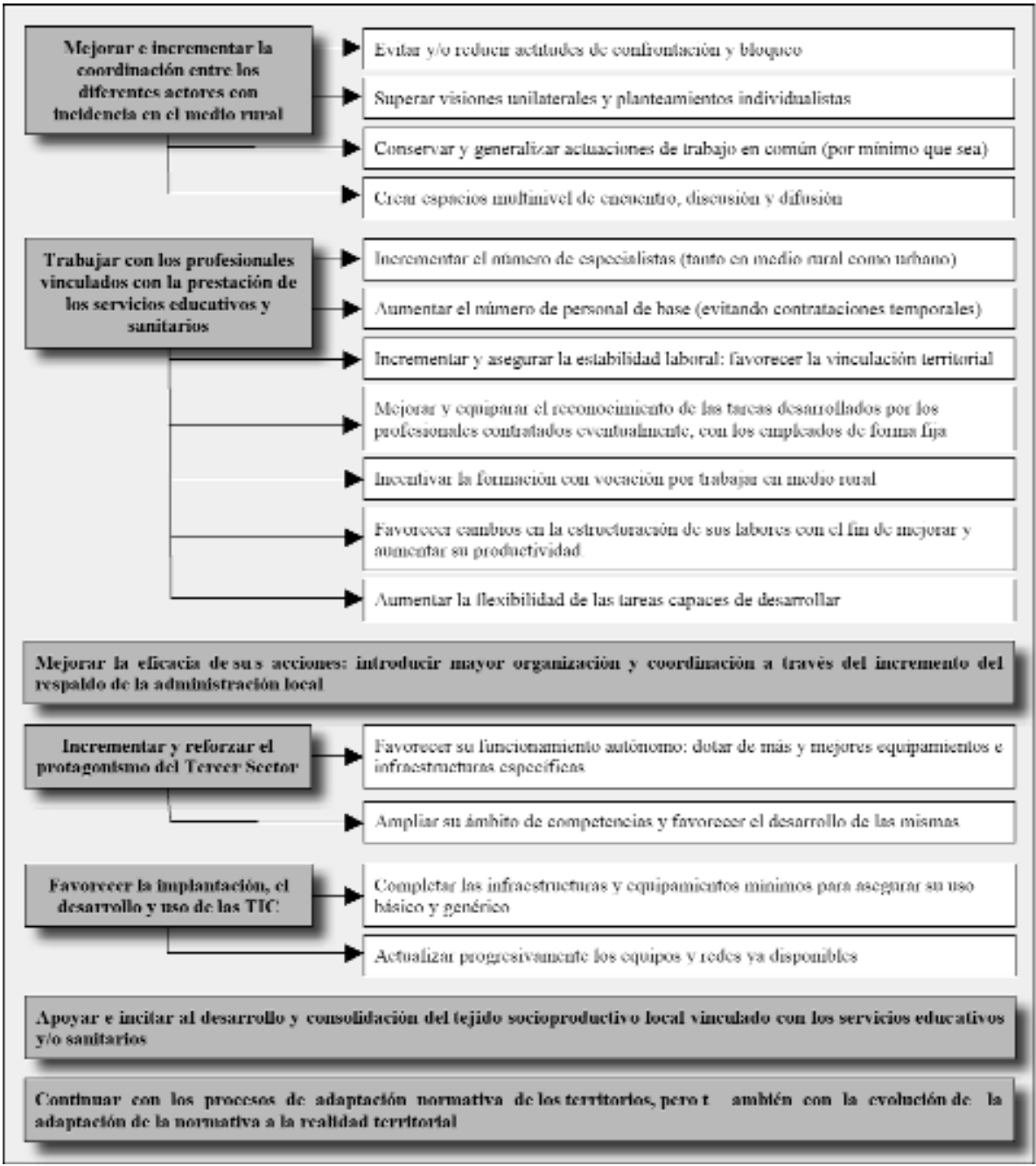

Fuente: Elaboración propia a partir de los resultados de las entrevistas realizadas a los representantes de la administración territorial, y de los servicios educativos y sanitarios seleccionados, 2006 y 2007.

vidades y programaciones conjuntas, evitar solapamientos y/o repeticiones, potenciar su posterior divulgación y conocimiento, etc. En definitiva, plantear una verdadera labor de coordinación y cooperación.

Una segunda agrupación de propuestas con la que mejorar el funcionamiento práctico de los servicios educativos y sanitarios, centra su ámbito de actuación sobre 
la figura de los profesionales encargados de gestionar y/o llevar a la práctica las atenciones ofertadas por estos servicios. Entre las sugerencias más repetidas destacan cuatro propuestas: aumentar el número de profesionales; facilitar la elección de los puestos de trabajo; mejorar la asignación de tareas en el espacio y/o en el tiempo; y permitir el desarrollo de todas sus funciones (sobre todo, aquéllas implícitas):

a) Cuando se señala el aumento de los profesionales, no sólo se hace referencia a los de carácter especializado, cuya escasez está ya en cierta forma asumida por la población local como un problema estructural. Al contrario, se piensa sobre todo en un aumento del personal encargado de desempeñar las acciones más básicas. En particular, porque la falta de este tipo de efectivos llega a ser tan importante como la otra. En consecuencia, con frecuencia se ha tomado como solución "temporal" la contratación de profesionales sanitarios extranjeros; pero ni la sociedad local ni los actores entrevistados ven en esta opción adecuada. Primero, por la "inestabilidad" de la medida, debido a que el problema continúa por el desajuste estructural entre oferta y demanda sanitaria. Y segundo, porque en ocasiones se presenta como una alternativa incluso contraproducente, debido a toda una serie de dificultades imprevistas que suelen aparecer y que afectan a la eficacia y eficiencia con que el servicio es prestado; por ejemplo, resultado de los problemas de comunicación entre los pacientes y los profesionales extranjeros contratados (en tanto en cuanto, algunos de ellos presentan un dominio del idioma local relativamente limitado).

Pero es que además, una fuerte contratación apenas planificada de profesionales extranjeros, puede provocar no sólo cierta deslegitimación del sistema, sino también problemas de inseguridad social. Sobre todo, si tenemos en cuenta la necesaria homogenización entre la formación de estos contratados y los títulos específicos presentes en territorio nacional, proceso que requiere bastante tiempo. En consecuencia, en ciertas ocasiones se contrata personal cuya formación profesional no se adecúa a las labores que tuviera que desempeñar.

En todo caso, el aumento del tamaño de las plantillas y en concreto del número de especialistas, no es una demanda única del medio rural. También es habitual de aquellas unidades de servicios emplazadas en zonas urbanas que se ven obligadas a que sus profesionales tengan que atender sus servicios especializados en áreas rurales contiguas. Aunque en realidad, los más perjudicados son los habitantes rurales, puesto que al ser éste un personal adscrito a varios establecimientos de forma simultánea, los centros rurales tienen que soportar a menudo la reducción de la atención "in situ" de estos profesionales, ante la obligación de responder en primer lugar a las necesidades de sus unidades urbanas de referencia. 
b) También vinculada a los profesionales, otra de las propuestas sugeridas se centra en asegurar e incrementar su estabilidad laboral a largo plazo, a través de una mayor facilidad en el acceso a los puestos de trabajo presentes en estas áreas para aquellos profesionales que los eligieran voluntariamente por motivos personales, residenciales y/o familiares. Esta idea se basa en el mismo sistema que otras comunidades emplean desde hace tiempo, mediante la creación de bolsas homologadas de trabajo específicas para territorios rurales (como por ejemplo, Cataluña). Así, se podría conseguir que los profesionales realmente interesados por trabajar en estas zonas pudieran hacerlo con relativa facilidad, y a la vez reducir la presencia de aquellos otros menos atraídos por estos destinos.

La creación de estas bolsas de empleo permitiría además, asegurar de un modo eficiente las sustituciones por enfermedad o descanso (es decir, en el menor tiempo posible y con el personal más adecuado para ello). En especial, si tenemos en cuenta la negativa recurrente del personal interino que, temporalmente sin empleo, se ve destinado a ocupar las vacantes que aparecen en zonas rurales. Esta situación se hace aún más evidente en los reemplazos de duración reducida (inferiores a un mes), debido a los esfuerzos extraprofesionales que este personal debe superar para desempeñar su labor (cuestiones de accesibilidad, dispersión de la demanda, menor dotación y actualización de recursos materiales, etc.), y que difícilmente se ven compensados en dichos períodos de tiempo (Díez, 1989).

Otras propuestas interesantes se centran en conseguir, por un lado, equiparar el reconocimiento de la labor desempeñada por parte de los sustitutos, con la de los profesionales fijos, mediante mejoras curriculares (aumento de la puntuación de cara a concursos de traslado) y/o retribuciones salariales acordes con el diferente contexto laboral. Y por otro, en aquellos casos en los que no hubiera otra solución, facilitar a la administración municipal la posibilidad de contratar directamente profesionales a cargo de sus recursos económicos.

c) Junto a los dos planteamientos previos, otro conjunto de mejoras a introducir vinculadas con los recursos humanos persigue facilitar cambios graduales en la organización temporal de los profesionales, siempre dentro de los marcos legales. La idea es que con mínimas readaptaciones en la distribución de los horarios de atención al público a la realidad rural, se puede incrementar la demanda atendida (y por tanto, ser más eficaz). De hecho, con frecuencia ésta está más determinada por costumbres y hábitos de vida en general, que por cuestiones de verdadera necesidad (por ejemplo, es habitual encontrar mayores solicitudes de atención médica los días de mercado municipal que el resto de la semana). Lógicamente, un mejor ajuste a la concentración real de las demandas, también permitiría ser más eficiente. 
d) La última actuación propuesta por los entrevistados para mejorar el funcionamiento de los servicios a través del personal contratado en ellos, se centra en la petición por incrementar su flexibilidad operativa sin por ello saturar su capacidad laboral. En especial, con el fin de poder abarcar así funciones no necesariamente propias, pero para las que sí se tuvieran competencias reconocidas, completando así sus tareas específicas con otras más generales; de este modo, y aunque fuera tan sólo temporalmente, se podrían suplir deficiencias en la cobertura elemental y/o especializada por falta de personal.

En tercer lugar, se recogen toda una serie de sugerencias centradas en la capacidad de actuación de la administración local, al ser ésta una de las principales responsables de la mejora de las prestaciones educativas y sanitarias si tenemos en cuenta, por un lado, la labor desempeñada como vía para evidenciar necesidades y/o insatisfacciones en relación a dichos servicios; y por otro, su papel como organismo responsable en ciertos casos de la resolución de dichas cuestiones, a través de la toma de medidas con que darles respuesta tanto de forma directa (al intervenir con recursos materiales, humanos, etc.) como indirecta (al incitar a la actuación de otras administraciones). De este modo, los entrevistados señalarán cuatro posibilidades:

a) Ante todo, la necesidad urgente de caracterizar al sistema público de una capacidad de respuesta ágil y flexible. En particular, para poder aprovechar positivamente la presencia de determinadas condiciones capaces de justificar y rentabilizar el desarrollo de inversiones con las que establecer, o simplemente ampliar, prestaciones que en otro contexto no se pudieran acometer (como es el caso de la demanda escolar de primaria, condicionada a una dinámica demográfica relativamente variable en estas zonas debido a continuos procesos migratorios).

Para ello, la reducción de los trámites administrativos a ejecutar se presenta como el principal mecanismo; es más, de este modo se lograría también mitigar en parte la dificultad que caracteriza el uso de determinados recursos especializados de los que disponen estos territorios, pero que por el momento debido a cuestiones burocráticas apenas son empleados (como por ejemplo, los sistemas de transporte aéreo medicalizado, o incluso los mismos profesionales, más destinados a elaborar informes que a mantener interacción directa con la población).

b) Disponer del personal especializado capaz, primero, de identificar adecuadamente las demandas de la población local; y segundo, con el suficiente conocimiento territorial como para encontrar y ofrecer las soluciones más adecuadas en función de los medios disponibles y actuaciones ya iniciadas (y evitar así la duplicación de esfuerzos y recursos). 
c) Ser congruente con la importancia que se le confieren a los servicios educativos y sanitarios, y dotarles de la representación adecuada en los órganos de gestión pública municipal. Sobre todo, en aquellos municipios con entidad suficiente para ello (por población, personal, competencias, etc.), en donde habría que evitar ocuparse de estos aspectos de forma combinada con otros (algunos de los cuales con aparentemente escasa relación); es decir, conceder el valor oportuno a ambos servicios mediante la creación de secciones concretas con personal capaz de dedicarle un mínimo de tiempo, de modo que se pudieran ofrecer respuestas oportunas, rápidas y coordinadas con otros colectivos e intereses.

d) Y por último, superar la cultura de la mera recepción de las subvenciones como medio para financiar y conseguir la realización de determinadas actividades (vinculadas directa o indirectamente con los servicios educativos y sanitarios, como por ejemplo, las actividades extraescolares llevadas a cabo por las AMPAS, etc.); para ello, se proponer instaurar en su lugar otro marco relacional, como por ejemplo los convenios de colaboración. En particular, porque de este modo se concibe la posibilidad de conseguir un tejido social más activo y comprometido de cara a completar las actividades regladas que educación y sanidad ofrecieran a sus poblaciones (Laguna, 2006); un aspecto que para los entrevistados no sólo puede aumentar la oferta de prestaciones, sino que también mejorar la calidad de las ya disponibles. Simplemente porque así se evitaría, a priori, que determinadas actuaciones se vieran interrumpidas en su ejecución ante la falta de dinero para hacer frente a la adquisición de materiales, y/o para pagar el salario de personal capaz de poner en marcha las actuaciones previstas.

Si en el conjunto anterior se incide en la necesidad de mejorar las actuaciones desarrolladas por parte de las administraciones locales, y se apunta en concreto la oportunidad de actuar sobre la relación de éstas con el tejido social formado por asociaciones y colectivos locales/regionales, un cuarto listado de sugerencias se agrupa específicamente sobre esta clase de agentes sociales. Sobre todo, debido a la amplitud y diversidad de ventajas que, según los entrevistados, la sociedad obtiene de la participación activa y decidida de estos actores sobre los servicios educativos y sanitarios (comentado ya anteriormente). De ahí que se incida con frecuencia en la conveniencia de favorecer su protagonismo, a partir de actuaciones concretas para mejorar la eficacia de sus actividades (al introducir una cierta organización y coordinación conjunta), favorecer su funcionamiento autónomo (dotándolas de equipamientos e infraestructuras suficientes y adecuadas), ampliar su ámbito de competencias (mediante su inclusión en la gestión y prestación de tareas concretas dentro de marcos de actuación más generales), etc. 
En quinto lugar, otro conjunto de soluciones para mejorar no sólo las prestaciones educativas y sanitarias de cara a la población, sino las condiciones laborales en las que muchos profesionales desarrollan sus tareas diarias, se centra en trabajar con las TIC. En especial, en los municipios con menor dotación en infoestructuras, en donde se hace necesario más que incorporar los últimos avances tecnológicos, acometer simplemente la dotación de los equipamientos informáticos elementales, y el desarrollo de conexiones básicas a las redes de telecomunicación (de telefonía móvil e internet): ordenadores, impresoras, faxes, instalación de antenas y/o repetidores de telefonía, etc. De este modo, se podría conseguir por ejemplo, que los profesionales empleados en los servicios de áreas rurales desarrollasen sus tareas en condiciones adecuadas; que mejorasen su formación, y por tanto afrontasen un conjunto de demandas relativamente más amplio y diverso (ante la posibilidad de seguir cursos on-line con los que actualizar conocimientos y metodologías); que aumentasen su productividad al permitir rentabilizar al máximo su limitada presencia, y favorecer actuaciones coordinadas entre varias unidades (como por ejemplo, la puesta en marcha de programaciones educativas y/o actuaciones en materia de salud sobre determinados segmentos de población); que rompieran la sensación de soledad presente en estas zonas al tener que trabajar de forma casi aislada, y conseguir así mejorar el atractivo de estos entornos laborales; etc.

Aunque también, disponer del equipamiento mínimo adecuado (y actualizado) beneficia a los propios usuarios de los servicios, al incrementar la facilidad de ser atendidos allí donde presentan sus demandas, gracias por ejemplo a la disponibilidad de acceder a su historia clínica sin tener que desplazarse a otros espacios y/o aplazar temporalmente sus peticiones. Pero también, es un hecho atractivo porque permite acceder a determinados servicios hasta hace poco tiempo inexistentes en estas zonas, o difíciles de conseguir con comodidad (por ejemplo, relacionados con la gestión de cuentas de ahorro, acceso a documentación necesaria para la realización de trámites administrativos con el sistema público -documentación necesaria para la matriculación, formularios para la solicitud de subvenciones-, etc.); etc.

La apuesta por conseguir un mejor y mayor aprovechamiento de las estructuras asistenciales, productivas y/o asociativas existentes en estos territorios constituye la sexta propuesta de los entrevistados. Por un lado, por la ineficacia de las iniciativas externas descontextualizadas de la realidad rural, y por tanto incapaces de atender las necesidades locales de forma adecuada. Un buen ejemplo, se encuentra en el "fracaso" del programa de comida a domicilio para personas mayores experimentado en ciertas localidades rurales; básicamente, porque en estos territorios esta población se muestra reacia a iniciativas que supongan la invasión de su espacio personal por desconocidos, aparte de que en ocasiones su cobertura temporal no es plena (sin servicio los festivos). 
Y por otro, porque atender a las necesidades locales con recursos de la zona permite convertir en oportunidades de empleo situaciones que en principio, podrían ser vistas como problemáticas (derivadas por ejemplo del envejecimiento demográfico y aumento de las demandas asistenciales). De este modo, se logra con frecuencia toda una serie de beneficios que convierten esta apuesta por lo local, en una de las vías de actuación más interesantes a fomentar por sus efectos "positivos" tanto en la economía como sobre la oferta laboral y la variedad atenciones prestadas.

En séptimo y último lugar, los entrevistados apuntaran igualmente otro tipo de acciones destinadas no tanto a mejorar las prestaciones de los servicios educativos y sanitarios ya disponibles en zonas rurales, sino a asegurar su funcionamiento y presencia. Para ello, se apunta como necesario dos líneas de trabajo: primero, continuar con el proceso de adaptación normativa de las legislaciones educativas y sanitarias a la realidad rural (por cuestiones de accesibilidad, dispersión de la demanda, escasa población, etc.), y cuyo mejor ejemplo hasta la fecha son los CRAS. Y segundo, que dicha proceso afecte de igual modo a aquellos aspectos normativos aprobados en anteriores ocasiones y en aplicación desde hace tiempo en estas áreas, pero que por evolución territorial pudieran haber quedado desfasados.

\section{Reflexiones finales}

En general, los problemas detectados se ajustan bien a la realidad que caracteriza al conjunto del mundo rural, y son formulados con bastante sentido común. No sólo porque superan las tradicionales demandas por conseguir todo tipo de servicios educativos y sanitarios en el ámbito local, sino también porque dejan de lado el empeño irracional por mantener físicamente operativos sus equipamientos e instalaciones en todo el espacio, por el simple hecho de que contar con ellas es positivo. Esta actitud, es sin duda consecuencia de la toma en conciencia por parte de la sociedad rural, de que su realidad territorial conlleva una especificidad diferente a la presente en zonas urbanas.

Por otro lado, el conjunto de soluciones propuestas por la sociedad rural para resolver dichos problemas y por tanto, permitir el uso eficiente y eficaz de los servicios educativos y sanitarios básicos por parte de la población local, e indirectamente aumentar las posibilidades de integrar sus equipamientos e instalaciones en los procesos de desarrollo rural, se caracterizan primero, por su proximidad y adecuación al territorio, puesto que inciden en aquellas cuestiones más frecuentes y comunes, y proponen en consecuencia actuaciones de ejecución desde y sobre los ámbitos locales. Y segundo, por su flexibilidad al ser capaces de actuar a la vez no sólo sobre problemas de diversa índole, sino también desarrollarse con frecuencia combinadas entre 
sí (y generar por consiguiente sinergias de resolución positivas). Sin embargo, al mismo tiempo cabe reconocer su relativamente limitada capacidad de cambio real, al apoyarse con frecuencia en modificaciones estructurales del comportamiento y organización de la sociedad, algo sin duda difícil de conseguir o cuanto al menos, costoso desde un punto de vista temporal.

Así, las soluciones propuestas en cuanto al desarrollo de espacios de cooperación mediante los que evitar actitudes de confrontación, son interesantes pero difíciles de llevar a cabo si tenemos en cuenta que habitualmente, cualquier propuesta de intervención sobre estos servicios no es considerada ni con la misma validez, ni con idéntica prioridad por los diferentes actores con intereses en ellos. De esta de manera, para aquéllos que parten de posiciones relativamente más favorables en cuanto a recursos, capacidad de movilización, contactos, etc., ven a priori más rápida y/o directamente recompensada su participación, que los que se sitúan en posiciones de mayor debilidad y/o aislamiento, al tener que esperar más tiempo para alcanzar los mismos objetivos, o cambiar la forma de evaluar los resultados (incidiendo por ejemplo en cuestiones más sociales que económicas, como crear empleo antes que generar riqueza); en consecuencia, la cooperación puede resultar "compleja" si este último conjunto de actores nunca ve atendidos sus intereses.

No obstante, consideramos que actuar de forma coordinada es una estrategia bastante oportuna si se quiere realmente abordar con éxito parte de los problemas detectados alrededor de ambos servicios; sobre todo, porque difícilmente de un trabajo sin conexión con el entorno, ni con el resto de niveles organizativos de un mismo sistema se pueden obtener resultados favorables de forma sostenible.

En cuanto a las diversas medidas propuestas por los entrevistados con incidencia particular sobre los profesionales, la más interesante es aquella que incide en la introducción y/o recuperación de una cierta multifuncionalidad del personal de base (de modo que así al menos pueden atenderse demandas que en la actualidad no se cubren por falta de profesionales específicos). No obstante, la aplicación de esta medida no debería en ningún caso prolongarse excesivamente en el tiempo; de ser así, la mayor parte de los recursos humanos elementales de que disponen estas zonas perderían tanto eficacia como eficiencia, al ver dificultadas la realización de sus tareas habituales por saturación de trabajo, y por posibles problemas de desmotivación.

Junto a ella, otra alternativa con posibilidades de conseguir resultados positivos es la dirigida a conseguir que los profesionales vinculados de manera previa con estos territorios, por los motivos que fuera, consigan emplearse en ellos. Una vía mucho más eficiente que la simple contratación general de personal, puesto que a pesar de que disponer de más trabajadores puede solucionar temporalmente problemas de falta de profesionales, la inestabilidad que actualmente caracteriza a estos supone al 
final no se consiga en realidad el objetivo perseguido (ya que para el paciente, la renovación constante de este personal supone la pérdida de la confianza necesaria para expresarse con seguridad y sentirse por tanto bien atendido).

De hecho, la inestabilidad que presentan los profesionales empleados en los servicios educativos y sanitarios de ámbito rural, tampoco encuentra solución adecuada a través del simple mantenimiento del actual sistema de recompensación laboral y/o profesional por trabajar en estos espacios. En concreto, por la mercantilización y/o banalización con que esta medida es observada en realidad, al concebirse tan sólo como una vía con la que conseguir rápidamente un beneficio profesional mediante el cual acceder a destinos con "mayor" atractivo. Lógicamente, la alternativa de generar en su lugar una cierta vocación por el medio rural, resulta al menos más interesante pero poco factible si tenemos en cuenta los valores y las modas que guían la sociedad actual.

Otra de las soluciones más atractivas es sin duda la apuesta por las TIC. No obstante, para ello es necesario que primero éstas se generalicen por el territorio y, segundo, que se lleve a cabo su completa actualización. De hecho, su impacto puede llegar a ser tan positivo que incluso con la actual disposición de recursos humanos se podría no sólo incrementar el tipo de prestaciones (no sería necesario que éstas se presentasen físicamente allí donde se localizase la demanda), sino también en muchos casos mejorar éstas (especialmente por la rapidez con que podrían conseguirse los resultados de cualquier prueba, el tratamiento de la información, etc.). Por ello, más que como una solución más al conjunto de propuestas realizadas, esta vía de trabajo debe observarse directamente como una línea estratégica de desarrollo obligado e incluso previo a otras alternativas. Aunque por el momento su potencialidad todavía se ve limitada para apostar únicamente por ella, puesto que primero se debe superar la brecha digital que aún caracteriza al espacio rural.

Por último, de las diversas propuestas presentadas en relación con la administración local, a pesar ser interesantes por enfatizar el papel desempeñado por dicho nivel y mostrar su capacidad para ayudar a resolver los problemas tanto de los servicios educativos como sanitarios (aun cuando este nivel apenas tuviera competencias directas en ninguno de ellos), su grado de realización (o al menos de permanencia) es relativamente limitado. Los escasos recursos materiales y humanos con que cuentan la mayoría de municipios rurales (en donde por ejemplo, es frecuente que ni tan siquiera el alcalde esté liberado de su trabajo para poder dedicarse plenamente a funciones de gestión local), supone que iniciativas de este tipo presenten con frecuencia problemas de sostenibilidad temporal. Para evitar esta situación, deberían conseguirse primero ciertos cambios estructurales en la organización de las administraciones, que permitieran a dicho nivel afrontar con garantía actuaciones en esta línea. 
En definitiva, todo el conjunto de situaciones problema enumeradas, y de soluciones adaptadas a las mismas (e incluso puestas en algunos casos en práctica), permiten comprender mejor la organización y disposición que los servicios educativos y sanitarios básicos adquieren habitualmente en las zonas rurales y, por tanto, la mayor o menor capacidad que los mismos tienen para influir en las dinámicas de desarrollo local. Lógicamente, porque no es lo mismo contar con un servicio activo de forma constante, con cierta cantidad y variedad de recursos, apoyado por los poderes públicos y complementado por la población local, que encontrarnos con una situación opuesta en donde la disponibilidad de las prestaciones está alejada y únicamente ofertada de forma puntual, apenas cuenta con el apoyo de la sociedad civil y que además, las diferentes administraciones con competencias son incapaces de ponerse de acuerdo sobre sus diferentes niveles de responsabilidad.

\section{Bibliografía}

Boix, R. (2003): "Escuela rural y territorio: entre la desruralización y la cultural local". En Revista Digital eRural, educación, cultura y desarrollo rural, 1(1), 8 pp.

Bourdieu, P. (1986): "The Forms of Capital". En Richardson, J. (Ed.): Handbook of theory and research for the Sociology of Education, Ed. Greenwood, New York, pp. 241-258.

Camarero, L. (coord.) (2009): La población rural de España: de los desequilibrios a la sostenibilidad social. Ed. Fundación "La Caixa”, Barcelona. 190 pp.

Díez, Mà.P. (1989): El profesor de E.G.B. en el medio rural. Universidad de Zaragoza, Zaragoza.

Entrena, F. (2006): "Difusión urbana y cambio social en los territorios rurales: un estudio de casos en la Provincia de Granada". En Revista de Estudios Regionales, 77, pp. 179-206.

Escalona, A y Díez C. (2007): "Despoblación territorial y oferta de servicios: diagnóstico y propuestas". En Serrano, C. (coord.) Despoblación y territorio. Zaragoza: Ed.
Centro de Estudios sobre la Despoblación y Desarrollo de Áreas Rurales, pp. 61-118.

Esparcia, J. (2010): "El potencial del análisis de contenidos como metodología para la caracterización de los modelos conceptuales-relacionales del desarrollo rural en España”. En Leco, F (coord.): Actas del XI Coloquio de Geografia Rural: Territorio, paisaje y patrimonio rural. Ed. Universidad de Extremadura y AGE, Cáceres.

Furuseth, O. (1998): "Service provision and social deprivation”. En Ilbery, B. (ed.): The geography of rural change. Ed. Longman, Londres, pp. 233-256.

Illeris, S. (2004): "Activités de services et développement régional. État de la recherche à partir de la littérature publiée entre 1998 et 2003". En Économies et Sociétés, 6, pp. 1833-1864.

Jean, Y. (1995): "École et aménagement du territoire rural: quel avenir pour les petites structures scolaires et les communes rurales?". En Annales de Géographie, 104 (583), pp. 236-255. 
Jean, Y. (dir.) (2007): Géographies de l'école rural: acteurs, réseaux, territoires. $\mathrm{Ed}$, Ophrys, París.

Laguna, M. (2006): "Más de veinte años de políticas de desarrollo rural en el Pirineo Aragonés". En Ager, Revista de Estudios sobre Despoblación y Desarrollo Rural, 5 , pp. $127-154$.

Melero, A. y Calatrava, A. (2003): "Procesos de terciarización en el medio rural". Sanz, J. (Coord.): El futuro del mundo rural: sostenibilidad, innovación y puesta en valor de los recursos locales. Madrid: Ed. Síntesis, pp. 73-101.

Morales, N. (2006): "Padres y profesores, dos importantes agentes sociales en las organizaciones escolares españolas y sus interrelaciones en un contexto glocal". En Convergencia. Revista de Ciencias Sociales, Universidad Autónoma del Estado de México, Toluca (México), pp. 87-116.

Moreno, A. y Escolano, S. (1992a): Los servicios y el territorio. Ed. Síntesis, Madrid.

Moreno, A. y Escolano, S. (1992b): "Los servicios colectivos para la población". En Moreno, A. y Escolano, S.: El comercio y los servicios para la producción y el consumo. Ed. Síntesis, Madrid, pp. 181-226.

Morente, M. (2004): "El cambio de lugar de trabajo". En Semergen, 30 (2), pp. 53-54.

Moyano, E. (2000): "Procesos de cambio en la sociedad rural española. Pluralidad de intereses en una nueva estructura de oportunidades". En Papers, 61, pp. 191-220.

Orriols, P.; Roldán, E. y Oliart, J. (2004): "Los servicios y actividades complementarias en la educación pública. Una aproximación empírica al papel de las asociaciones de madres y padres de alumnos en Cataluña”. En Índice, 18, pp. 2.
Peraldi, X. y Pieri, X. (2006): "Services de santé, territoires ruraux et gouvernance territoriale: quelques enseignements de la situation de la Corse". En XVI International RESER Conference, Lisboa. Services Gouvernance and Public Policies. 37 pp. En <http://www.reser.net/file/ 28487/>. [17 de marzo de 2011].

Poirey, J.L. (2006): "Réussite scolaire au collège et implication des acteurs : l'action au carrefour des territoires et des réseaux". En Alpe, Y., Champollion, P. y Poirey, J.L. (coords.): L'enseignement scolaire en milieu rural et montagnard. Le devenir des élèves en fin de Collège: parcours et projets. Vol. 4, Ed. Presses Universitaires de France-Comté, Besançon, pp. 121-126.

Rodríguez González, R. (1999): "Los servicios en el espacio rural”. En Antón Burgos, F.J. (coord.): La geografia de los servicios en España, Madrid: Ed. Asociación de Geógrafos Españoles, pp. 164-174.

Romero, J. y Farinós, J. (2007): "Los territorios rurales desvitalizados en el cambio de siglo". En Rubio Terrado, P. y Sanz, A. (coords.): Investigación aplicada al desarrollo de territorios rurales frágiles. Zaragoza: Ed. Prensas Universitarias de Zaragoza, pp. 131-147.

Rubio, R. (2003): "Medicina rural: una tradición renovada". En Sesenta y más, 222, pp. 46-51.

Saco, A. (2010): "Desarrollo rural y despoblación en Galicia: escenarios y desarrollos de futuro". En Ager, Revista de Estudios sobre Despoblación y Desarrollo Rural, 9, pp. 11-30.

Santamaría, R. (1999): La educación secundaria en zonas rurales. Documento de análisis del CEFIRE de Castellón, extensión Segorbe (Castellón), 22 pp. En $<$ http://intercentres.cult.gva.es/cefire/1240 0780>. [17 de marzo de 2011]. 
Subirats, J. (2002): "Educació i comunitat". En Temps d'educacio, 26, pp. 181-200.

Tickell, A. (1999): "The geographies of services: new wine in old bottles". En Progress in Human Geography, 4 (23), pp. 633-639.

Tickell, A. (2002): "Geography of services: progress in the geography of services III time to move on?". En Progress in Human Geography, 26 (26), pp. 791-801.

Valles, M. (2002): Entrevistas cualitativas. Ed. Centros de Investigaciones Sociológicas. Serie Cuadernos Metodológicos, 32. Madrid. 